\title{
Early radiological worsening of SARS-CoV-2 pneumonia predicts the need for ventilatory support
}

\author{
Juana María Plasencia-Martínez ${ }^{1} \cdot$ Andrés Carrillo-Alcaraz $^{2} \cdot$ Miguel Martín-Cascón $^{3} \cdot$ Rafael Pérez-Costa $^{4}$. \\ Mónica Ballesta-Ruiz ${ }^{5}$. Ana Blanco-Barrio ${ }^{1}$ - Ignacio Herves-Escobedo ${ }^{1}$. José-Miguel Gómez-Verdú ${ }^{3}$. \\ Julián Alcaraz-Martínez ${ }^{4} \cdot$ Sergio Alemán-Belando ${ }^{3} \cdot$ María José Carrillo-Burgos $^{4}$
}

Received: 9 August 2021 / Revised: 16 October 2021 / Accepted: 18 October 2021 / Published online: 16 January 2022

(c) The Author(s), under exclusive licence to European Society of Radiology 2021

\begin{abstract}
Objectives Identifying early markers of poor prognosis of coronavirus disease 2019 (COVID-19) is mandatory. Our purpose is to analyze by chest radiography if rapid worsening of COVID-19 pneumonia in the initial days has predictive value for ventilatory support (VS) need.

Methods Ambispective observational ethically approved study in COVID-19 pneumonia inpatients, validated in a second outpatient sample. Brixia score (BS) was applied to the first and second chest radiography required for suspected COVID-19 pneumonia to determine the predictive capacity of BS worsening for VS need. Intraclass correlation coefficient (ICC) was previously analyzed among three radiologists. Sensitivity, specificity, likelihood ratios, AUC, and odds ratio were calculated using ROC curves and binary logistic regression analysis. A value of $p<.05$ was considered statistically significant.

Results A total of 120 inpatients ( $55 \pm 14$ years, 68 men) and 112 outpatients ( $56 \pm 13$ years, 61 men) were recruited. The average ICC of the BS was between 0.812 (95\% confidence interval $0.745-0.878$ ) and 0.906 (95\% confidence interval 0.844-0.940). According to the multivariate analysis, a BS worsening per day $>1.3$ points within 10 days of the onset of symptoms doubles the risk for requiring VS in inpatients and 5 times in outpatients $(p<.001)$. The findings from the second chest radiography were always better predictors of VS requirement than those from the first one.

Conclusion The early radiological worsening of SARS-CoV-2 pneumonia after symptoms onset is a determining factor of the final prognosis. In elderly patients with some comorbidity and pneumonia, a 48-72-h follow-up radiograph is recommended.

Key Points

- An early worsening on chest X-ray in patients with SARS-CoV-2 pneumonia is highly predictive of the need for ventilatory support.

- This radiological worsening rate can be easily assessed by comparing the first and the second chest $X$-ray.

- In elderly patients with some comorbidity and SARS-CoV-2 pneumonia, close early radiological follow-up is recommended.
\end{abstract}

Keywords COVID-19 $\cdot$ Radiography $\cdot$ Prognosis $\cdot$ Mechanical ventilation $\cdot$ Brixia score

Abbreviations
$\begin{array}{llll}\text { BS } & \text { Brixia score } & \text { d-OS } & \text { Days from onset of the symptoms } \\ \text { COVID-19 } & \text { Coronavirus disease 2019 } & \text { RT-PCR } & \begin{array}{l}\text { Real-time reverse transcription polymer- } \\ \text { ase chain reaction }\end{array} \\ \text { CXR } & \text { Chest X-ray } & & \text { S }\end{array}$

Juana María Plasencia-Martínez plasen79@gmail.com

1 Radiology Department, Hospital General Universitario JM Morales Meseguer, Avenida Marqués de los Vélez, s/n, 30008 Murcia, Spain

2 Intensive Care Medicine Department, Hospital General Universitario JM Morales Meseguer, Avenida Marqués de los Vélez, s/n, 30008 Murcia, Spain
3 Internal Medicine Department, Hospital General Universitario JM Morales Meseguer, Avenida Marqués de los Vélez, s/n, 30008 Murcia, Spain

4 Emergency Medicine Department, Hospital General Universitario JM Morales Meseguer, Avenida Marqués de los Vélez, s/n, 30008 Murcia, Spain

5 Epidemology and Public Health Regional Health Council, IMIB-Arrixaca, Universidad de Murcia, Murcia, Spain 
SARS-CoV-2 Severe acute respiratory syndrome coronavirus 2

VS

WRDR
Ventilatory support

Worsening radiological day rate

\section{Introduction}

The high level of contagiousness of SARS-CoV-2 has overwhelmed many health systems in few months, especially intensive care units (ICU). COVID-19 mainly affects the respiratory system and can cause serious lung damage leading to hypoxemic acute respiratory failure and even acute respiratory distress syndrome $[1,2]$. Current treatment is based on corticosteroids and respiratory support, ranging from basic oxygen therapy to extracorporeal oxygenation. The abrupt clinical worsening that occurs about a week from the onset of symptoms can have an outcome ranging from complete recovery to death. Risk factors for poor prognosis, especially the need for hospital admission and ventilatory support, have been analyzed in multiple studies [3, 4]. But it remains unclear what factors determine these differences in the course of the disease and, above all, what markers we can use to anticipate irreversible clinical deterioration. Research related to radiological prognostic markers has mainly focused on the first chest radiograph (CXR) findings [5-8]. However, there are few studies have used interval chest radiographs for prognostication of the disease [9].

Our objective is to analyze if rapid worsening of COVID19 pneumonia in the initial days has predictive value for the need of ventilatory support (VS), regardless of it consisted in non-invasive ventilation or endotracheal intubation, given the overlap between these two forms of therapy based on physicians' experience. Our hypothesis is this evolution may provide a more robust prognostic information than that provided by the initial radiograph alone.

\section{Methods}

Observational ambispective single-center study approved by our institutional ethics committee (internal code EST: 38/20), following the STROBE Statement. Patient informed consent was waived.

\section{Setting and sample selection}

We recruited a sample of COVID-19 inpatients from March 14th to April 17th, 2020 (sample 1, S1). If our hypothesis was confirmed in S1, it would be validated in a second sample of COVID-19 outpatients, recruited from October 27th, 2020, to January 25th, 2021 (sample 2, S2).

The eligibility criteria for both samples were as follows: patients aged 18-80 years; SARS-CoV-2 infection confirmed by RT-PCR, antibody, or antigen testing; unfavorable respiratory course; and CXR lung opacities by probable or indeterminate COVID-19 pneumonia [10, 11]. For S1, when eligible, patients were admitted and included. Additionally, patients were also admitted when clinical suspicion of pneumonia was high and they had risk criteria for pneumonia progression (i.e., age $\geq 60$ or comorbidities; Suppl-Fig. 1a), even when a first CXR did not show signs of pneumonia. For S2, patients were consecutive included when they did not have admission criteria at the time of their first CXR (i.e., no dyspnea, respiratory rate $<22$, oxygen saturation $\geq 95 \%$, $\mathrm{BS}<14$, and no risk of progression). However, they needed further assessment and a second CXR due to an unfavorable clinical course while isolated at home the month after the first CXR (hereinafter, "reconsultation") (Suppl-Fig. 1b).

SARS-CoV-2 infection was confirmed by the primary care physician before requesting the CXR1 or by the emergency physician shortly after the CXR1. ICU admission was determined by the severity of the respiratory failure and the need for VS (Suppl-Table 1). Patients older than 80 years were not eligible because they could not be considered candidates for VS in extreme resource-constrained situations.

Patients (a) with non-diagnostic radiological images, due to poor technical quality, and (b) referrals from other hospitals admitted to the ICU, due to the lack of previous data were excluded (Suppl-Fig. 1).

\section{Radiological variables}

To determine the radiological evolution, data from CXR1 and CXR2 were collected. CXR1 was considered the 1st CXR performed during hospital admission for COVID-19 for sample S1, or the 1st CXR showing lung opacities probable or indeterminate for COVID-19 pneumonia [10] for sample S2. CXR2 was the first follow-up CXR after CXR1 in both samples.

CXRs were always reported by a radiologist or radiology resident.

For CXR1 in both samples and for CXR2 in S2, standard posteroanterior thorax view was assessed, and for nonmobile patients, bedside portable radiography.

For CXR2 in S1, portable radiography was always used to reduce the in-hospital contagion risk.

The severity of pulmonary involvement was quantified using the Brixia score (BS) [11] on the basis of the posteroanterior or anteroposterior CXR projection. The BS was chosen because it is specific to COVID-19, and, in our opinion, more straightforward to calculate than other scores (e.g., RALE) as it does not measure the extent per lung zone BS ranges between 0 (absence of involvement) and 18 points (maximum total involvement, 3 points 


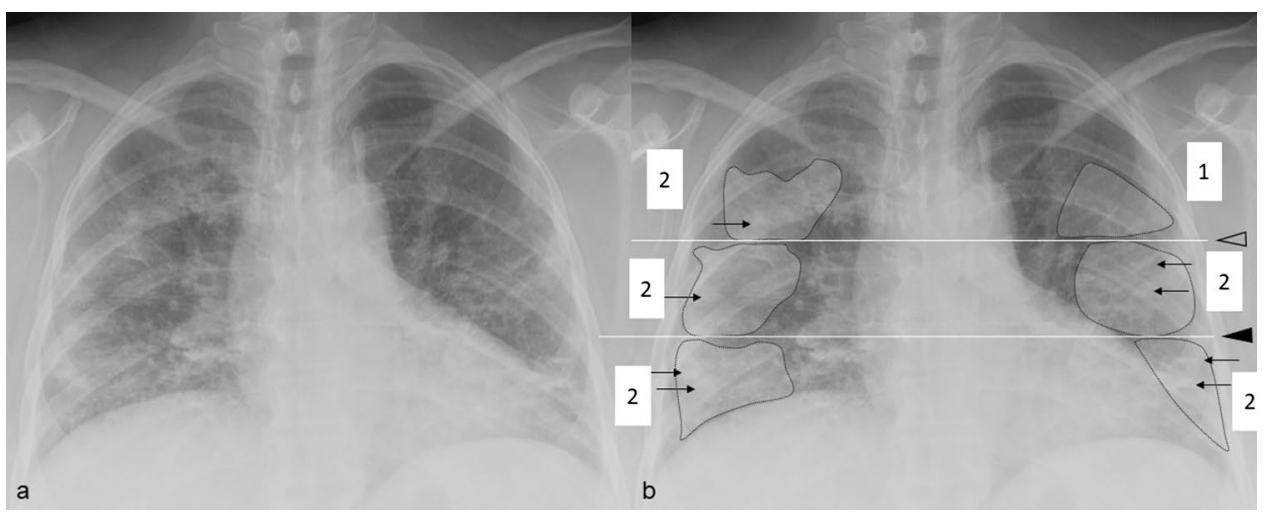

Fig. 1 Example Brixia score (BS) measurement in supine chest X-ray (CXR), in patient with dyspnea, SARS-CoV-2 infection confirmed by RT-PCR and pneumonia. a Original radiograph. b Original radiograph with reference marks for BS calculation: the lung fields are divided by drawing a horizontal line below the aortic arch (hollow arrowhead) and another dividing the visible lung parenchyma into two parts of equal height down to the lowest diaphragm below the

maximum per lung zone, 6 zones, Fig. 1). BS scores on CXR1 and CXR2 were denominated Brixia score 1 (BS1) and 2 (BS2), respectively. Severity and evolution of lung involvement in each lung zone were assessed by the BS mean value and its percentage of change ((BS2meanBS1mean) $* 100 / 3)$.

Two radiologists (observer 1, 8-year experience in thoracic and emergency radiology and observer 2, 8-year experience in emergency radiology) and a third-year resident (observer 3) retrospectively calculated the BS1 in $\mathrm{S} 1$, blinded to observer 1 and 2, the CXR2 or any clinical data. Previous radiological studies could be consulted to detect preexisting abnormalities. To analyze the concordance of BS in supine radiography studies, observers 1 and 3 calculated BS2 in a sample obtained by simple randomization of 50 patients from among all S1 patients undergoing a second portable radiograph as CXR2. Once tested the BS reproducibility, data obtained for observer 1 was used for S1 analysis; after workshops had been conducted for all the radiologists involved, the BS was prospectively calculated and used for decision-making and for analysis in S2 (Suppl-Table 1). The degree of experience in reading CXR among radiologists and residents in-training for S2 ranged between 2 and 22 years. Although experience is variable among radiologists, all CXRs are reported in our hospital during on-call duty, so the radiologist is used to reading it.

For standardizing the BS worsening, we defined the worsening radiological day rate (WRDR) as (BS2-BS1)/ difference of days between CXR1 and CXR2, resulting in the daily increase in BS. A positive WRDR value indicated worsening, and a negative one, radiological improvement. first line (black arrowhead). Ground glass opacities (GGO) have been drawn with a dotted margin area while black arrows point to small consolidations. In BS, opacities are quantified as 0: normal; 1: GGO; 2: GGO and consolidations with GGO predominance; 3: consolidations with or without GGO, with consolidation predominance. In the example a, 2 points were assigned to all zones, excepting the left superior zone with 1 point, with a BS of 11 points

\section{Non-radiological variables}

The main endpoint was the VS need (request criteria in Suppl-Table 1). We also collected sex, age, comorbidities, chronic treatment with immunosuppressants and antihypertensive drugs, symptoms, need and length for hospitalization and ICU, days from onset of the symptoms (d-OS), corticosteroid treatment, $\mathrm{SpO} 2 / \mathrm{FiO} 2$ and inflammatory markers levels related to the episode of COVID-19.

To determine whether early radiological follow-up would be feasible in patients most likely to require VS, we defined "vulnerable population," in the absence of specific published criteria, as those patients with at least one comorbidity and older than 55 years.

\section{Statistical methods}

S1 size corresponded to all eligible first wave COVID-19 inpatients. Based on $\mathrm{S} 1$ results, assuming a proportion of VS requirement of $17 \%$, a precision of $6.5 \%$, and a need for replacement rate of $10 \%$, the S2 size considering $95 \%$ confidence was estimated to be 109 patients.

Quantitative variables were expressed as means \pm standard deviation or median (interquartile range, IQR) according to the normal or non-normal distribution, and qualitative variables using absolute and relative frequencies. $\mathrm{Chi}^{2}$, Mann-Whitney $U$, Kruskal-Wallis, Student $T$, and Spearman correlation tests were used for bivariate comparison. The reading agreement was evaluated by intraclass correlation coefficient (ICC) and Bland-Altman tests.

Radiological variables performance was determined using the maximum value of the Youden index in the 
receiver operating characteristic curve, with values of area under the curve (AUC), sensitivity, specificity, and positive (PLR) and negative (NLR) likelihood ratios with the 95\% confidence interval (95\% CI).

The multivariate analysis by binary logistic regression was performed to determine the association between VS need and the main effects of non-radiological variables.

Differences were considered statistically significant at $p<0.05$. IBM-SPSS-Statistics version 20, Med-Calc version 12.7.0, JASP 0.14.0.0, and Excel-MicrosoftOffice-365 software were used. Analysis was performed by JPMP (radiologist) and MBR (statistician).

\section{Results}

\section{Sample description}

S1 consisted of 120 hospitalized patients $(55 \pm 14$ years, $68 \mathrm{men}), 26(21.7 \%)$ of them requiring VS (Fig. 2a, Table 1). The most frequent symptoms were fever (temperature $>37.5^{\circ} \mathrm{C}, 74.2 \%$ ), cough $(70 \%)$, and dyspnea $(40 \%)$.

The median time between the onset of the symptoms and CXR1 was 7 days (5-11).

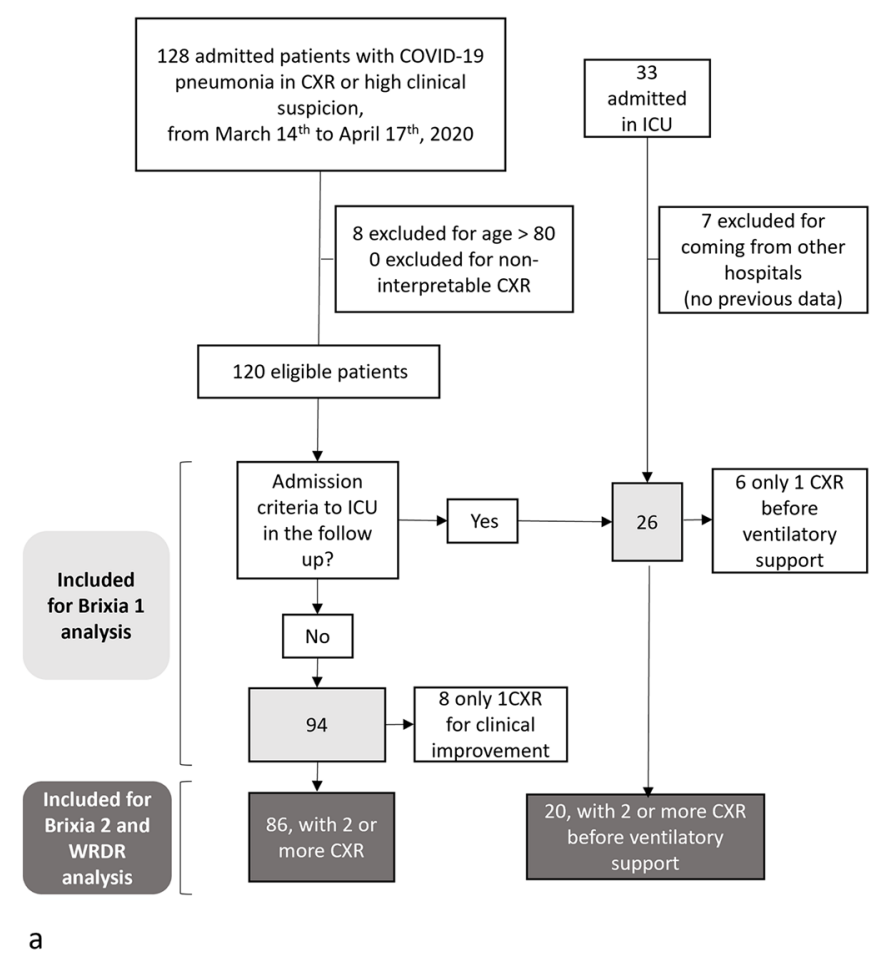

S2 consisted of 112 patients who reconsulted (56 \pm 13 years, $61 \mathrm{men}), 65$ (58\%) of whom required hospitalization and 14 (12.5\%) VS (Fig. 2b, Table 2).

\section{Radiological variables}

The average ICC between observers $1-2,1-3$, and $2-3$ for the BS1 was 0.906 (95\% CI 0.844-0.940), 0.812 (95\% CI $0.745-0.878$ ), and 0.878 (95\% CI 0.823-0.916), respectively. The mean of the differences in the Bland-Altman test was $0.7(95 \% \mathrm{CI}-3.0 ; 4.5), 0.8(95 \% \mathrm{CI}-4.4 ; 6)$, and 0.1 $(95 \% \mathrm{CI}-4.5 ; 4.7)$, respectively. The average ICC between observers $1-3$ for the BS2, assessed from portable radiography, was 0.905 (95\% CI 0.833-0.946), and the mean of the differences in the Bland-Altman test was 0.3 (95\% CI-4.7; 5.3). The medians of BS1, BS2, and WRDR were 5 (3-8), 5 (2-9), and $0.18(-1 ; 1)$ in $S 1$, and $4(3-6), 7(4-0)$, and 0.5 $(0 ; 1)$ in $\mathrm{S} 2$. The days between CXRs varied less in S1 $(2$ (2-2) vs $5(3-6)$ ), as it was established by protocol only for S1 (Suppl-Table 1; Tables 1 and 2). The first follow-up CXR and the first VS device were required 3 and $4 \mathrm{~d}-\mathrm{OS}$, and at 3 and $6 \mathrm{~d}-\mathrm{OS}$ for $\mathrm{S} 1$ and S2, respectively.

In $\mathrm{S} 1$, lung opacities were present in the right middle (RMZ 82\%), right lower zone (RLZ 72\%), left lower (65\%) and left middle (63\%) zones in CXR1. Severity followed the same distribution (mean BS1 $=1.23,1.17,1.04$, and 1.01). Radiological severity peaked in the lower zones

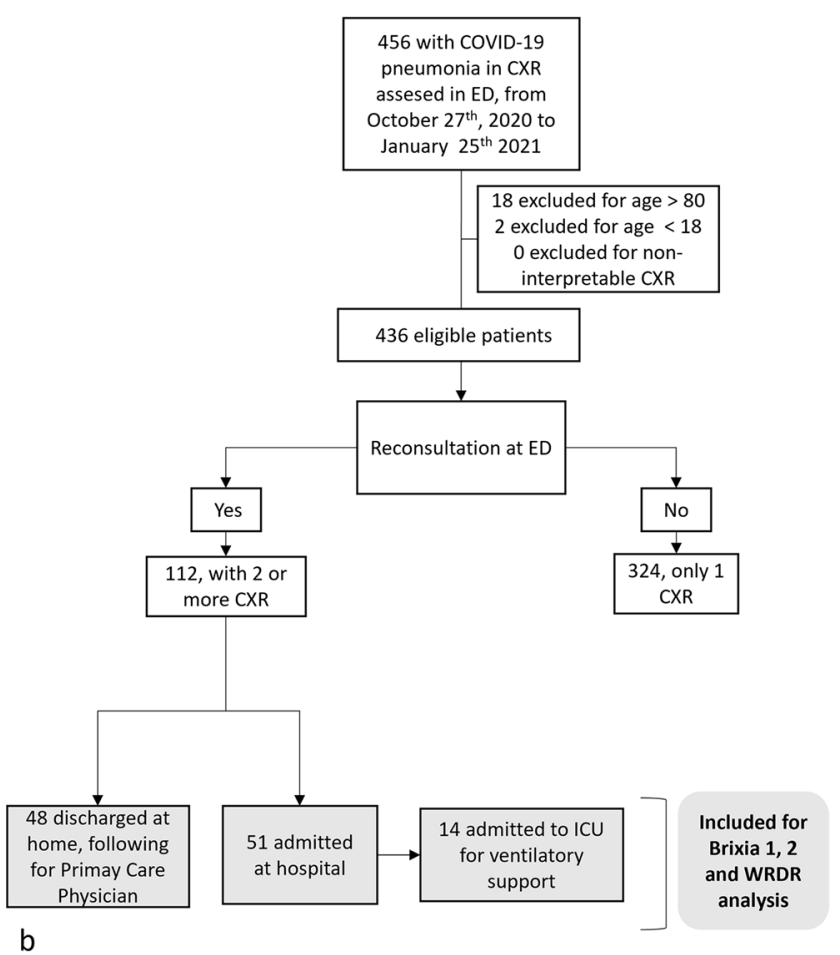

Fig. 2 Flow chart of patients included and excluded in samples 1 (a) and 2 (b). Abbreviations: CXR, chest X-ray; ED, emergency department; ICU, intensive care unit; WRDR, worsening radiological day rate 
Table 1 Characteristics of sample 1

\begin{tabular}{|c|c|c|c|c|}
\hline & $\begin{array}{l}\text { All } \\
(n=120)\end{array}$ & $\begin{array}{l}\text { No ventilatory support } \\
(n=94)\end{array}$ & $\begin{array}{l}\text { Ventilatory support } \\
(n=26)\end{array}$ & $p^{*}$ \\
\hline Gender, male & $68(56.7 \%)$ & $49(52.1 \%)$ & $19(73.1 \%)$ & .06 \\
\hline Age & $55.07 \pm 13.84$ & $53.83 \pm 14.01$ & $59.54 \pm 12.42$ & .06 \\
\hline Comorbidities & $45(37.5 \%)$ & $39(41.5 \%)$ & $6(23.1 \%)$ & .09 \\
\hline -Hypertension & $48(40 \%)$ & $36 / 94$ & 12 & .47 \\
\hline -Diabetes mellitus & $18(15 \%)$ & $11(11.7 \%)$ & $7(26.6 \%)$ & .05 \\
\hline -Active or previous smoking & $29(24.2 \%)$ & $23(24.5 \%)$ & $6(23.1 \%)$ & .88 \\
\hline -Chronic pneumopathy & $22(18.3 \%)$ & $17(18.1 \%)$ & $5(19.2 \%)$ & .89 \\
\hline -Cancer & $4(3.3 \%)$ & $2(2.1 \%)$ & $2(7.7 \%)$ & .16 \\
\hline -Cardiovascular or cerebrovascular disease & $16(13.3 \%)$ & $10(10.6 \%)$ & $6(23.1 \%)$ & 1 \\
\hline -Chronic treatment with immunosuppressants & $4(3.3 \%)$ & $3(3.2 \%)$ & $1(3.8 \%)$ & .87 \\
\hline $\begin{array}{l}\text {-Antihypertensive (Angiotensin Converting Enzyme } \\
\text { inhibitors or Angiotensin II Receptor Antagonists) } \\
\text { therapy }\end{array}$ & $34(28.3 \%)$ & $21(22.3 \%)$ & $13(50 \%)$ & .006 \\
\hline -Others & $13(10.8 \%)$ & $1(1.1 \%)$ & $12(46.2 \%)$ & $<.001$ \\
\hline Methylprednisolone pulses & $36(30 \%)$ & 30/94 (31.91\%) & $6 / 26(23.07 \%)$ & .38 \\
\hline $\mathrm{SpO} 2 / \mathrm{FiO} 2$ & $400(272.92-461.90)$ & $442.86(332.14-461.90)$ & $226.25(153.57-299.14)$ & $<.001$ \\
\hline Days from OS to hospitalization & $7(5-11)$ & $9(6-12)$ & $6(4-9)$ & .02 \\
\hline Days from OS to $1^{\text {st }} \mathrm{CXR}$ & $5(7-11)$ & $8(6-12)$ & $6(3.75-8.25)$ & .008 \\
\hline Days from OS to $2^{\text {nd }} \mathrm{CXR}^{* *}$ & $7(10-14)$ & $10(8.5-14)$ & $8(6-9.75)$ & .001 \\
\hline Days between $1^{\text {st }} \mathrm{CXR}$ and $2^{\text {nd }} \mathrm{CXR} * *$ & $2(2-2)$ & $2(2-2.25)$ & $2(1-2)$ & .01 \\
\hline Brixia score 1 & 5 (3 to 8$)$ & $5(3-7)$ & $8.5(4.75-11.25)$ & .001 \\
\hline Brixia score $2 * *$ & $5(2$ to 9$)$ & $4(2-6.2)$ & $12(9-14)$ & $<.001$ \\
\hline Worsening radiological day rate ${ }^{* *}$ & $0.18(-1$ to 1$)$ & $-0.37(-1$ to 0.14$)$ & $2.5(1.68$ to 4.88$)$ & $<.001$ \\
\hline
\end{tabular}

Results are shown as absolute frequency (percentage), mean \pm standard deviation if normal distribution, and median (25 and 75 quartiles) if no normal distribution. Ventilatory support means non-invasive ventilation or endotracheal intubation requirements

*Mann-Whitney or chi ${ }^{2}$ tests

*** Data obtained from patients who underwent the 2nd CXR: $n=86$ and 20 with no ventilatory support and with ventilatory support

Abbreviations: $C X R$, chest X-ray; $O S$, onset of symptoms

and the RMZ within $5 \mathrm{~d}-\mathrm{OS}$, and in the left middle zone, between 5 and $10 \mathrm{~d}-\mathrm{OS}$. Upper zone involvement was less frequent and milder, especially on the left (54\% and $45 \%$, mean BS $1=0.72$ and 0.63 , respectively) and occurred later (at 10-15 d-OS, Suppl-Table 2a).

In patients with VS requirement, BS1 was significantly greater only in $\mathrm{RMZ}$ (mean $\mathrm{BS}=1.55$ vs $1.12, p=0.043$ ); however, BS2 was greater in all zones $(p \leq 0.042)$, mainly in RLZ (33.33\%) and RMZ (26.67\%), excepting left upper zone $(p=0.05)$. When VS was not required, BS2 decrease respecting to BS1 in all zones between 0.39 and $7.36 \%$, although it was only significant for lower zones and right upper zone ( $p \leq 0.048$, Suppl-Table 2b).

In $\mathrm{S} 1$, radiological severity was higher in men in CXR1 (BS1 of $6.5 \pm 3.9$ vs $4.9 \pm 3.4 ; p=0.02$ ), and it tended to be so in CXR2 (BS2 of $6.5 \pm 4.7$ vs $4.8 \pm 4.0 ; p=0.07$ ). Patients with rapid radiological worsening were admitted earlier (rho between d-OS to admission with BS2 and WRDR $=-0.226$ and $-0.243 ; p<0.005)$. However, the initial radiological severity was not decisive (rho with BS1 $=-0.088$ ).
BS2 correlated better with $\mathrm{SpO} 2 / \mathrm{FiO} 2$ and systemic inflammatory markers levels (rho range $=0.174$ and -0.576 ) than BS1 (rho range $=0.125$ and -0.493 ), except for ferritin and D-dimer levels on admission, with better correlation for BS1 (Suppl-Table 3).

According to the multivariate analysis (Table 3), the predictive ability for VS need was always worse for BS1 than for BS2 and WRDR. A two-unit increase of BS1 within 10 d-OS raised the risk of VS [95\% CI] in 0.9 times ([0.7-1.1], $p=0.20, \mathrm{AUC}=0.635$ [0.502-0.754]) in $\mathrm{S} 1$, and 1.7 times $([1.2-2.6] ; p=0.005, \mathrm{AUC}=0.557[0.506-0.608])$ in $\mathrm{S} 2$. However, a two-unit increase of BS2 incremented the risk 2.4 times ([1.7-3.4], $p<0.001, \mathrm{AUC}=0.931$ [0.835-0.980]) in $\mathrm{S} 1$, and 2.4 times ([1.7-3.3], $p<0.001, \mathrm{AUC}=0.812$ [0.688-0.903], Table 4) in S2. Patients who required VS always showed higher BS2, and BS1 was higher only for the bivariant analysis in S1 (Fig. 3). Patients with the highest BS1 (rho $=-0.567, p=0.003$ ) were admitted earlier to ICU, and this correlation was weaker for BS2 (rho $=-0.446$, $p=0.06$ ). 
Table 2 Characteristics of sample 2

\begin{tabular}{|c|c|c|c|c|c|}
\hline & $\begin{array}{l}\text { Reconsultation } \\
(n=112)\end{array}$ & $\begin{array}{l}\text { Reconsultation } \\
\text { and new discharge } \\
(n=48)\end{array}$ & $\begin{array}{l}\text { Reconsultation } \\
\text { and hospitalization } \\
(n=51)\end{array}$ & $\begin{array}{l}\text { Reconsultation and } \\
\text { ventilatory support } \\
(n=14)\end{array}$ & $p^{*}$ \\
\hline Gender, male & $61(54.5 \%)$ & $24(50 \%)$ & $28(54.9 \%)$ & $9(64.3 \%)$ & .68 \\
\hline Age & $56.07 \pm 13.02$ & $53.35 \pm 13.76$ & $56.63 \pm 12.31$ & $64.00 \pm 9.41$ & .02 \\
\hline Comorbidities & $27(24.1 \%)$ & $8(16.7 \%)$ & $12(23.5 \%)$ & $7(50 \%)$ & .04 \\
\hline -Diabetes mellitus & $9(8 \%)$ & $2(4.2 \%)$ & $6(11.8 \%)$ & $1(7.1 \%)$ & .38 \\
\hline -Cardiovascular disease & $11(9.8 \%)$ & $11(3.4 \%)$ & $2(4.2 \%)$ & $5(9.8 \%)$ & .02 \\
\hline -Neurologic disease & $2(1.8 \%)$ & $5(1.5 \%)$ & $1(2.1 \%)$ & $1(7.1 \%)$ & .20 \\
\hline -Chronic pneumopathy & $17(15.2 \%)$ & $6(12.5 \%)$ & $7(13.7 \%)$ & $4(28.6 \%)$ & .31 \\
\hline -Chronic treatment with immunosuppressants & $6(5.4 \%)$ & $3(6.3 \%)$ & $3(5.9 \%)$ & $0(0 \%)$ & .64 \\
\hline -Dexamethasone therapy & $81(72.3 \%)$ & $37(77.1 \%)$ & $34(66.7 \%)$ & $11(78.6 \%)$ & .47 \\
\hline Days from OS to $1^{\text {st }} \mathrm{CXR}$ & $5(3-7)$ & $6(3-8)$ & $6(4-7)$ & $5(3-5.25)$ & .31 \\
\hline Days from OS to $2^{\text {nd }} \mathrm{CXR}$ & $10(8-13)$ & $11(9-16)$ & $10(8-12)$ & $8.5(6-10)$ & .008 \\
\hline Days between $1^{\text {st }} \mathrm{CXR}$ and $2^{\text {nd }} \mathrm{CXR}$ & $5(3-6)$ & $6(4-9)$ & $4(3-6)$ & $3.5(2.7-5.2)$ & .007 \\
\hline Score Brixia 1 & $4(3-6)$ & $3.5(2.25-5)$ & $5(2-6)$ & $4.5(3-7)$ & .17 \\
\hline Score Brixia 2 & $7(4-10)$ & $4(3.6)$ & $8(6-10)$ & $10(9-13)$ & $<.001$ \\
\hline Worsening radiological day rate & $0.5(0$ to 1$)$ & $0(-1.7$ to 0.5$)$ & $0.75(0.25$ to 1.13$)$ & $2(0.92$ to 2.7$)$ & $<.001$ \\
\hline
\end{tabular}

Results are shown as absolute frequency (percentage), mean \pm standard deviation if normal distribution, and median (25 and 75 quartiles) if no normal distribution. Ventilatory support means non-invasive ventilation or endotracheal intubation requirements. ${ }^{*} \mathrm{Kruskal}^{-W a l l i s}$ or chi ${ }^{2}$ tests

The increase in radiological severity in conjunction with the early request for a second CXR (Tables 1 and 2) in patients with VS requirement was highlighted by the WRDR. A WRDR $>1.3$ within 10 d-OS multiplied the probability of needing VS [95\% CI] by $2.0([1.3-3.2], p<0.001)$ in $\mathrm{S} 1$ and by 5.0 ([2.2-11.4], $p<0.001$, Table 3) in S2, with sensitivity, specificity, PLR, NLR, and AUC values of 94\% [70-100], 93\% [82-99], 14.06 [4.7-42.3], 0.067 [0.01-0.4], and 0.903 [0.800-0.964] in S1, and of 83\% [52-98], 87\% [74-95], 6.39 [2.9-14.0], 0.19 [0.05-0.7], and 0.867 [0.752-0.942] in S2 (Table 4). In 24/26 S1 patients (92\%) with VS requirement, WRDR was $>1$ (median 2.5, IQR 1.68-4.88) in the 0-10 d-OS. In 92/94 patients (98\%) without VS requirement, WRDR was $<1$ in S1 during the entire follow-up period. These patterns were replicated in S2. The WRDR differences were evident as early as $0-5 \mathrm{~d}-\mathrm{OS}$, with WRDR median (IQR) of $2.5(1.75 ; 5.5)$ vs $-0.50(-1.5 ; 0.0 ; p<0.001)$ for VS requirement in $\mathrm{S} 1$, and of $2.33(0.67 ; 3.00), 0.7(0.17$; $1.22)$, and $0.33(-0.11 ; 0.62)$ for VS requirement, hospitalization vs discharge in S2 $(p<0.001$, Fig. 4$)$.

\section{Non-radiological variables}

In $S 2$, patients older $(64 \pm 9,57 \pm 12$ vs $53 \pm 14$ years; $p=0.02)$ and with at least one comorbidity (7 (50\%), 12 $(23.5 \%)$, vs $8(16.7 \%) ; p=0.04$, Table 2$)$ required more support (VS, hospitalization vs discharge). In S1, patients treated with antihypertensive drugs $(13(50 \%)$ vs $21(22 \%)$ $p=0.006)$, with diabetes mellitus (7 (26.6\%) vs $11(11.7 \%)$; $p=0.05)$, previous cardiovascular or cerebrovascular disease (6 (23.1\%) vs $10(10.6 \%) ; p=0.09)$, and males (19 (73.1\%) vs $49(52.1 \%) ; p=0.06$, Table 1$)$ tending to need more VS.

If early radiological follow-up had been performed in vulnerable patients or with pneumonia on the first CXR with a BS $>4$ (since this is the threshold determined by the Youden point on the ROC curve that best defines the need for VS on S1), 212/456 (46.5\%) patients older than 55, 76/456 (16.7\%) with some comorbidity and 273/456 (59.9\%) with a BS1 > 4 would have undergone follow-up CXR among the total number of patients presenting with pneumonia between October 27th, 2020, and January 25th, 2021 (Fig. 2). Considering the day with the most reconsultations (13/01/21), 19 follow-up CXRs would have been requested: 14 (over 55 years) plus 1 (some comorbidity) plus 4 (BS1 $>4)$.

\section{Discussion}

The early changes of pulmonary opacities assessed in the CXR are a determining factor in the clinical evolution of patients with COVID-19 pneumonia. A worsening of Brixia score $>1.3$ points per day within 10 days after onset symptoms increases the VS need twofold in inpatients, and five times in outpatients.

The prognostic role of imaging techniques in patients with SARS-CoV-2 pneumonia has been focused on the first CXR [6-8, 12]. However, the predictive ability of the lung involvement assessed by BS on the second CXR was better in our study, probably because this follow-up better depicts 


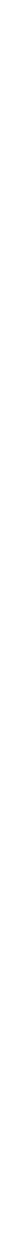

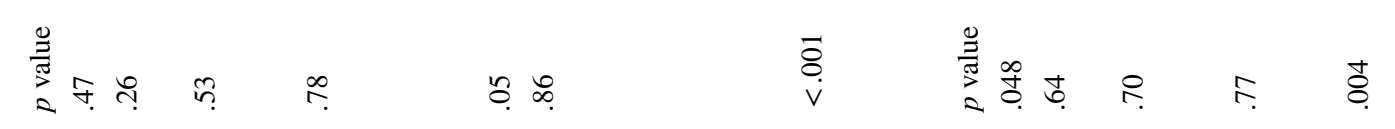

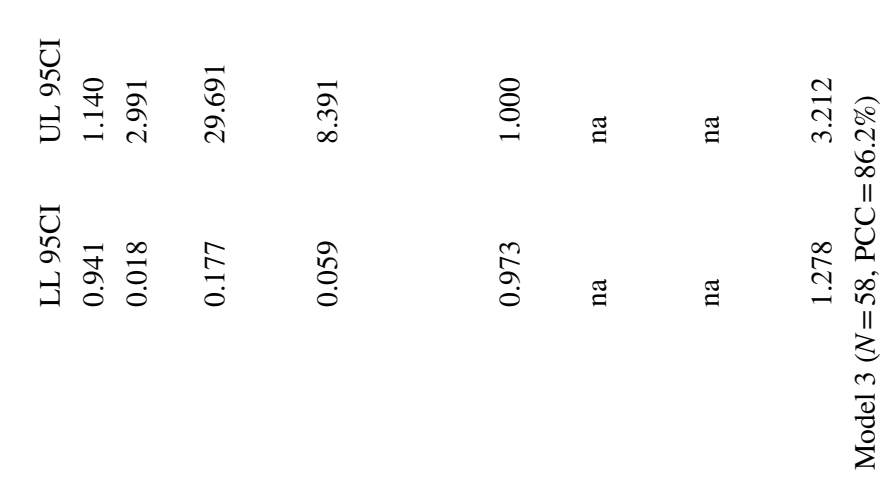

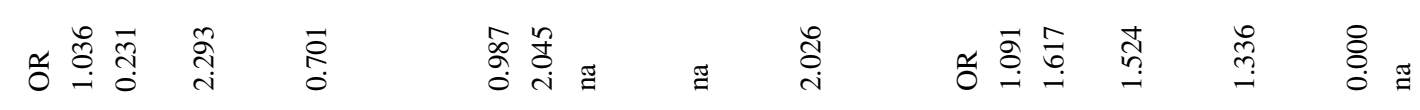

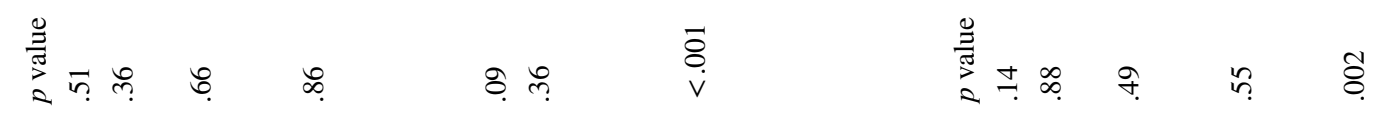

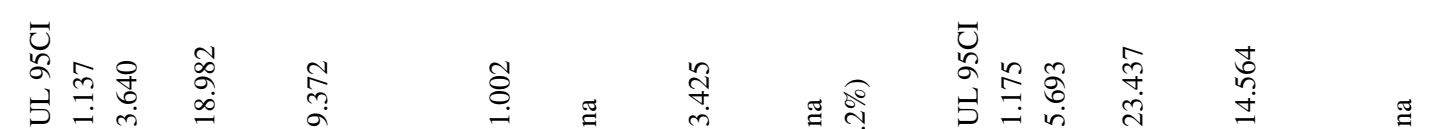

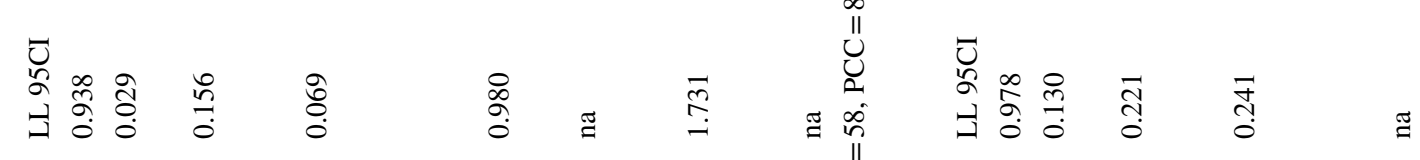

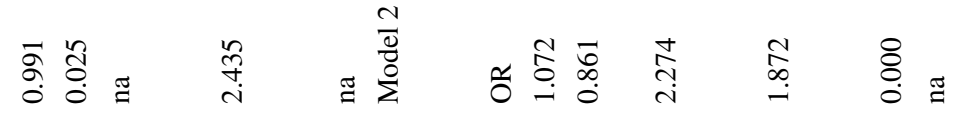

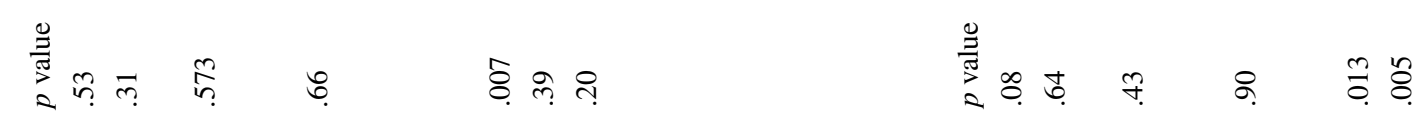

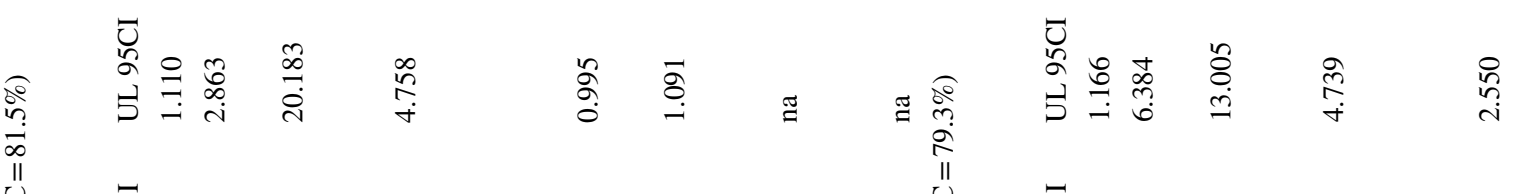

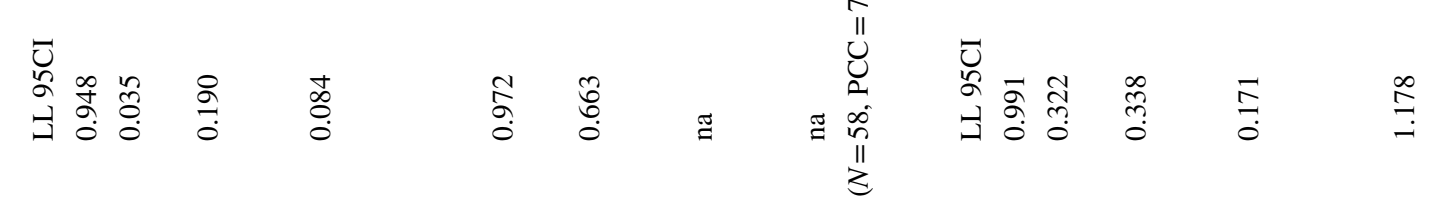

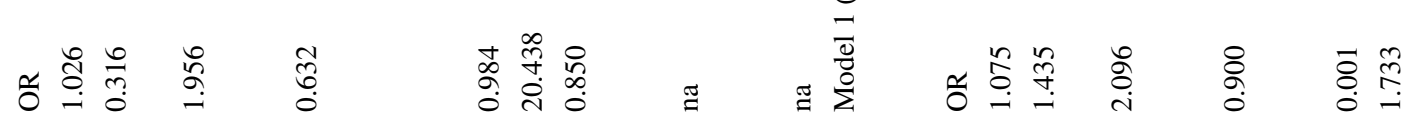

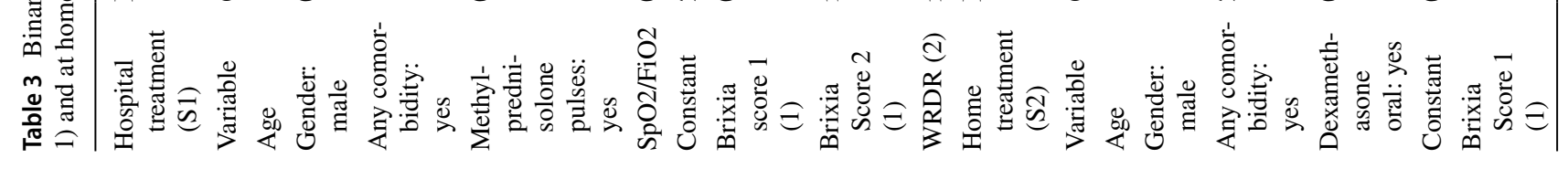


the enormous capacity of COVID-19 for a rapid clinical worsening. BS evaluated in the second CXR was better correlated with systemic inflammatory markers and $\mathrm{SpO} 2 / \mathrm{FIO} 2$ than in the first CXR. The worsening radiological per day rate, calculated by dividing the increase of the BS between two consecutive CXR by the number of days between both exams, is our best parameter for characterizing the SARS$\mathrm{CoV}-2$ pneumonia progression. Therefore, not only radiological involvement, but also the speed at which changes occur, standardizing per day, prevents the influence that the different time elapsed between the first and second CXR may have on the radiological changes. For example, an increase in BS greater than 4 points between two radiographs 3 days apart would mean a daily increase of Brixia score $>1.3$. We could also extrapolate this worsening rate by dividing the BS in whatever CXR performed within the first 10 days of symptoms by the days from symptom onset to CXR performing date. For example, a BS of 10 points in a CXR on the fourth day since symptom onset, assuming pneumonia onset on the first day of symptoms, would mean a daily increase in BS of 2.5 points ( $>1.3$ per day), or even higher if pneumonia onset were later. Both scenarios would imply a high probability of requiring VS. However, given the high reproducibility for the CXR findings [13], the radiological changes between radiographs are probably a more objective measure of evolution than the patient's perception of the days since the onset of symptoms and the assumption of the day that the pneumonia begins. A follow-up with a second CXR can provide an inexpensive and more simple marker of rapid disease progression risk than other complex prognostic models or algorithms [7, 14], warning of the need of ICU admission, preventing delays establishing VS treatment to stabilize respiratory failure, and possibly avoiding endotracheal intubation and death. Awaiting evidence-based guidelines, our recommendation for patients with COVID-19 pneumonia and BS $>4$ is to perform a follow-up CXR [9, 15] after 48-72 h, given that VS may be required as quickly as 4 days from the onset of symptoms, at least in patients older than 55 or with some comorbidity. This following-up workload had been sustainable in our environment (19 CXRs in the busiest day). Males, elderly, and patients with some comorbidity (requiring antihypertensive therapy, diabetics, and with cardio- or cerebrovascular disease) were our most vulnerable patients, like previous studies also suggest [16].

COVID-19 pneumonia evolves in extensiveness and severity following a distribution, starting in the right middle and lower zones, with progression towards the left middle zone, and finally the upper zones, at 5, 5-10, and 10-15 day of onset symptoms, respectively, as has been previously reported [17].

Having analyzed two samples could be considered a limitation; however, it has allowed us to validate our hypothesis, making the findings more robust and generalizable. 
Table 4 Diagnosis performance of Brixia score 1 and 2 for ventilatory support

\begin{tabular}{|c|c|c|c|c|c|c|}
\hline & AUC & Threshold & Sensitivity & Specificity & PLR & NLR \\
\hline BS1-S1 & $\begin{array}{l}0.635 \\
{[0.502 \text { to } 0.754]}\end{array}$ & $>7$ & $15 / 26(59)[36.4-79.3]$ & 76/94 (81) [68.0-90.6] & $59 / 19(3.13)[1.6-6.0]$ & $41 / 81(0.50)[0.3-0.8]$ \\
\hline BS1-S2 & $\begin{array}{l}0.557 \\
{[0.506 \text { to } 0.608]}\end{array}$ & $>4$ & $7 / 14(50)$ [21.1-78.9] & $55 / 99$ (56) [50.5-60.8] & $50 / 44(1.13)[0.6-2.0]$ & $50 / 56(0.90)[0.5-1.6]$ \\
\hline BS2-S1 & $\begin{array}{l}0.931 \\
{[0.835 \text { to } 0.980]}\end{array}$ & $>7$ & $24 / 26$ (94) [69.8-99.8] & $75 / 94$ (80) [65.4-90.4] & $94 / 20$ (4.69) [2.6-8.5] & $6 / 80(0.08)[0.01-0.5]$ \\
\hline BS2-S2 & $\begin{array}{l}0.812 \\
{[0.688 \text { to } 0.903]}\end{array}$ & $>8$ & $13 / 14(92)$ [61.5-99.8] & $67 / 99$ (67) [52.0-80.5] & $92 / 33(2.81)[1.8-4.4]$ & $8 / 67(0.12)[0.02-0.8]$ \\
\hline WRDR-S1 & $\begin{array}{l}0.903 \\
{[0.800 \text { to } 0.964]}\end{array}$ & $>1.3$ & $24 / 26$ (94) [69.8-99.8] & 88/94 (93) [81.7-98.6] & $94 / 7$ (14.06) [4.7-42.3] & 6/93 (0.07) [0.01-0.4] \\
\hline WRDR-S2 & $\begin{array}{l}0.867 \\
{[0.752 \text { to } 0.942]}\end{array}$ & $>1.3$ & $12 / 14(83)$ [51.6-97.9] & $86 / 99$ (87) [73.7-95.1] & $83 / 13$ (6.39) [2.9-14.0] & $17 / 87(0.19)[0.05-0.7]$ \\
\hline
\end{tabular}

Data are numerator/denominator unless otherwise indicated, data in parentheses are percentages, and data in brackets are $95 \%$ confidence intervals

Abbreviations: $B S 1$, Brixia score 1; BS2, Brixia score 2; $N L R$, negative likelihood ratio; $P L R$, positive likelihood ratio; $S 1$, sample 1; S2, sample 2; WRDR, worsening radiological day rate

The exclusion of patients older than 80 years constitutes another limitation; however, having included patients in this age range could have possibly entailed a more relevant bias, as they would not have received VS in a resource-scarce scenario even when clinically indicated. Future studies will be required to replicate the results in this population. The variable obesity was not collected in the part of the sample collected retrospectively; however, this limitation has no impact on the most relevant findings of the work. Finally, although a beneficial effect of corticosteroid treatment on VS requirements has not been highlighted, specifically focused studies on this point are required. Nonetheless, we believe that the radiological prognostic ability is not affected by this factor.

Summarizing, a daily increase of the Brixia score $>1.3$ points at onset of SARS-CoV-2 pneumonia raises the possibility of requiring VS. We recommend radiological
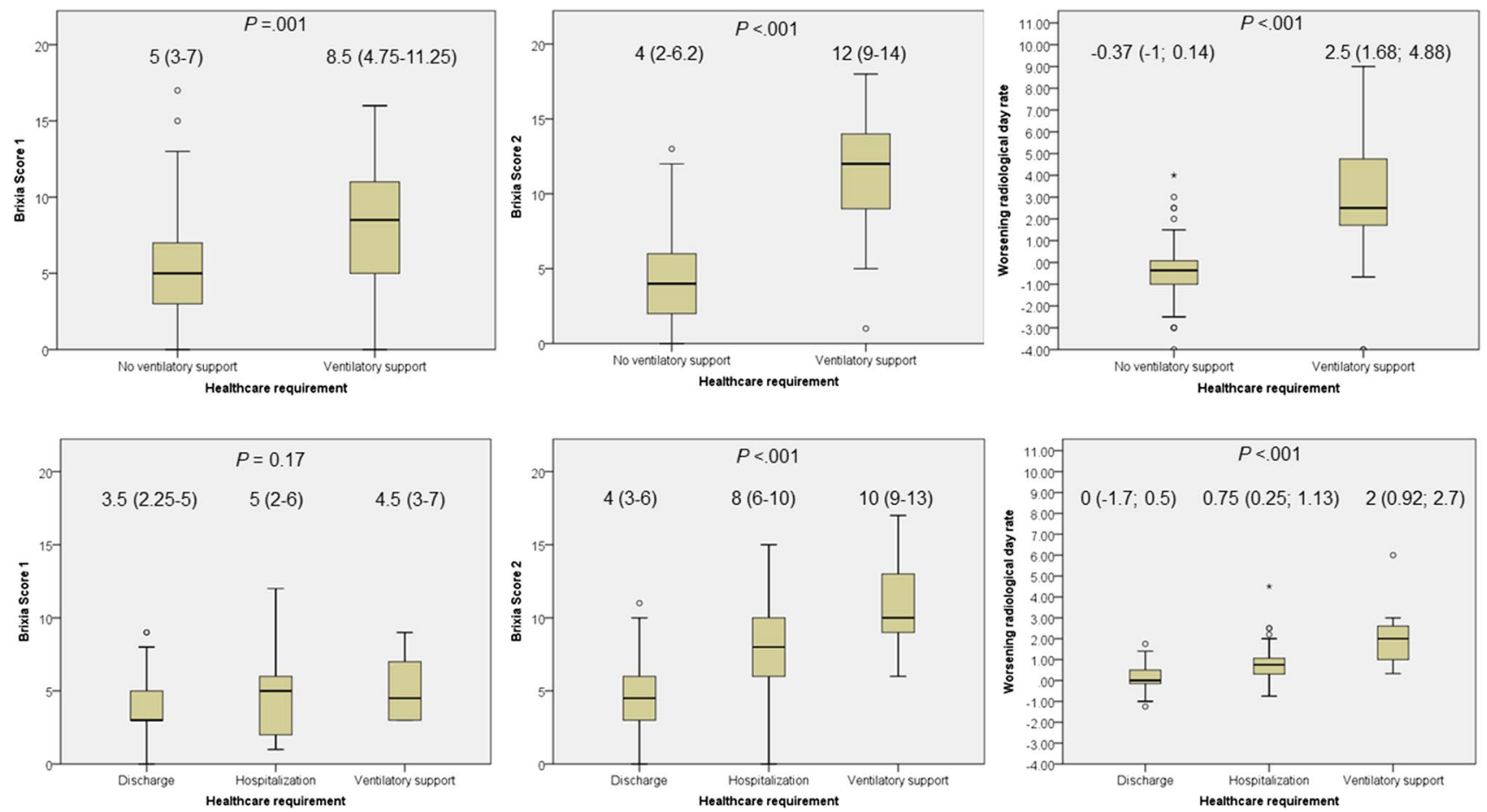

Fig. 3 Differences in Brixia score 1 and Brixia score 2. Worsening radiological day rate in patients according to the healthcare requirements in patients treated in hospital (sample 1, upper row), and at home (sample 2, lower row). For each variable, the median (interquartile range) is shown; $n$ is shown in Tables 1 and 2. The $p$-value has been calculated by Mann-Whitney $U$ or Kruskal-Wallis test 


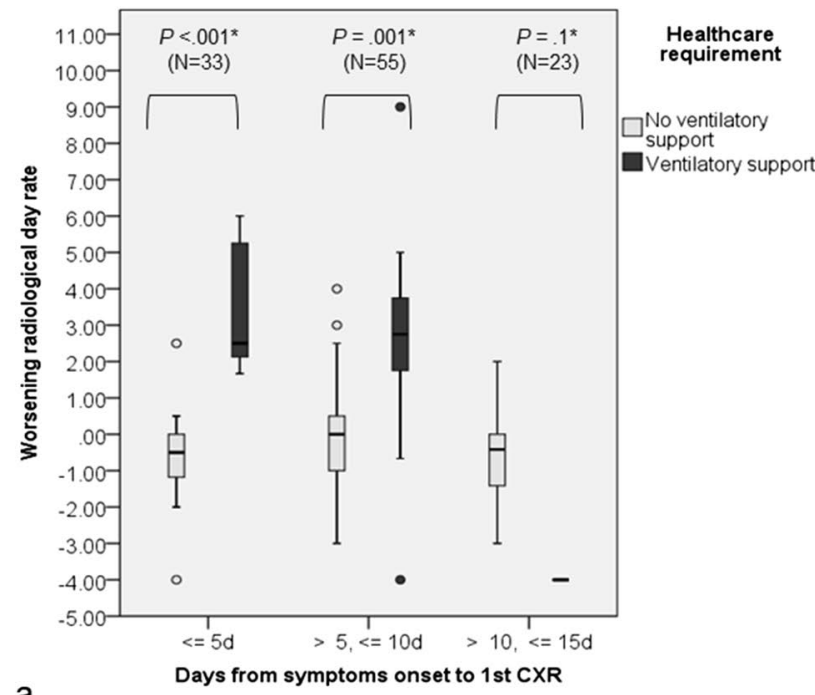

a

Fig. 4 Differences in worsening radiological day rate in patients with and without respiratory support requirement compared to the days from the onset of the symptoms to the 1st CXR in samples 1 (a) and 2 (b). The values for each variable are shown in the text and in

follow-up 48-72 $\mathrm{h}$ after the first CXR with findings of pneumonia, at least in elderly patients with some comorbidity.

Supplementary Information The online version contains supplementary material available at https://doi.org/10.1007/s00330-021-08418-3.

Acknowledgements We are grateful to Dr García Santos for his contribution to this manuscript.

\section{Declarations}

Guarantor The scientific guarantor of this publication is Juana María Plasencia Martínez.

Conflict of interest JMPM is member of the European Radiology Editorial Board. She has not taken part in the review or selection process of this article. The authors of this manuscript declare no relationships with any companies, whose products or services may be related to the subject matter of the article.

Statistics and biometry Mónica Ballesta Ruiz, statistician, has significant statistical expertise. She is also one of the authors of the manuscript.

Informed consent Written informed consent was waived by the Institutional Review Board.

Ethical approval Institutional Review Board approval was obtained.

\section{Methodology}

- prospective and retrospective

- cross-sectional study/prognostic study/observational

- performed at one institution

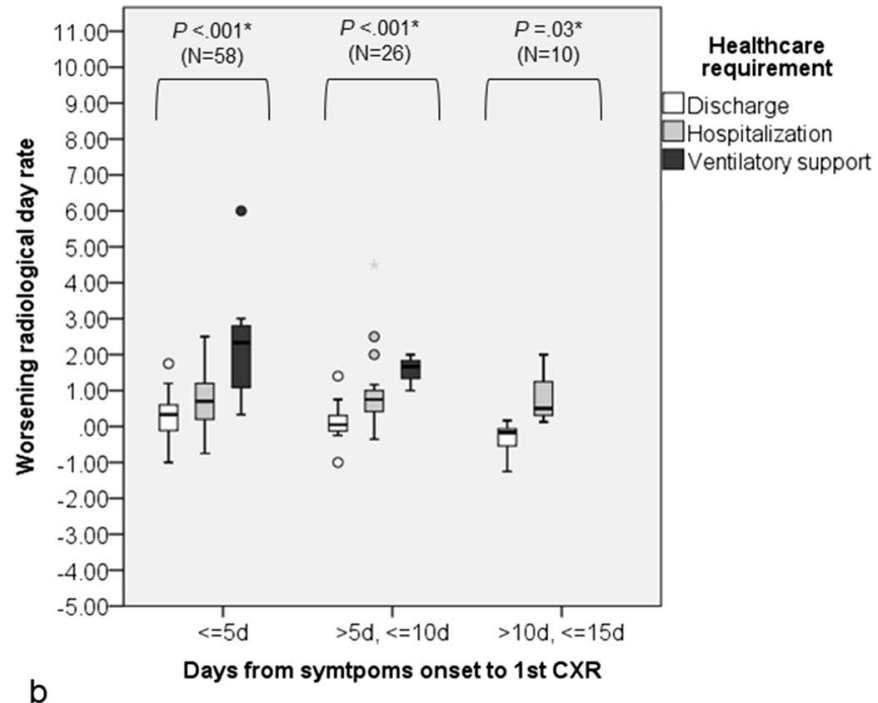

Tables 1 and 2. The $p$-value has been calculated by Mann-Whitney $U$ test or Kruskal-Wallis test. Abbreviations: CXR, chest X-ray; d, days; VS, ventilatory support

\section{References}

1. CDC (2020) Healthcare Workers. In: Centers for Disease Control and Prevention. https://www.cdc.gov/coronavirus/2019-ncov/ hcp/clinical-guidance-management-patients.html. Accessed 6 Apr 2021

2. Yang X, Yu Y, Xu J et al (2020) Clinical course and outcomes of critically ill patients with SARS-CoV-2 pneumonia in Wuhan, China: a single-centered, retrospective, observational study. Lancet Respir Med 8:475-481. https://doi.org/10.1016/S22132600(20)30079-5

3. CDC (2020) COVID-19 and Your Health. In: Centers for Disease Control and Prevention. https://www.cdc.gov/coronavirus/2019ncov/need-extra-precautions/people-with-medical-conditions. html. Accessed 6 Apr 2021

4. Huang I, Lim MA, Pranata R (2020) Diabetes mellitus is associated with increased mortality and severity of disease in COVID19 pneumonia - a systematic review, meta-analysis, and metaregression. Diabetes Metab Syndr 14:395-403. https://doi.org/10. 1016/j.dsx.2020.04.018

5. Xiao N, Cooper JG, Godbe JM et al (2021) Chest radiograph at admission predicts early intubation among inpatient COVID19 patients. Eur Radiol 31:2825-2832. https://doi.org/10.1007/ s00330-020-07354-y

6. Reeves RA, Pomeranz C, Gomella AA et al (2020) Performance of a severity score on admission chest radiograph in predicting clinical outcomes in hospitalized patients with coronavirus disease (COVID-19). AJR Am J Roentgenol. https://doi.org/10.2214/AJR. 20.24801

7. Mushtaq J, Pennella R, Lavalle S et al (2021) Initial chest radiographs and artificial intelligence (AI) predict clinical outcomes in COVID-19 patients: analysis of 697 Italian patients. Eur Radiol 31:1770-1779. https://doi.org/10.1007/s00330-020-07269-8

8. Pagano A, Finkelstein M, Overbey J et al (2021) Portable chest radiography as an exclusionary test for adverse clinical outcomes during the coronavirus disease 2019 pandemic. Chest. https://doi. org/10.1016/j.chest.2021.01.053 
9. Maroldi R, Rondi P, Agazzi GM et al (2020) Which role for chest $\mathrm{X}$-ray score in predicting the outcome in COVID-19 pneumonia? Eur Radiol. https://doi.org/10.1007/s00330-020-07504-2

10. COVID-19 BSTI Reporting templates I The British Society of Thoracic Imaging. https://www.bsti.org.uk/covid-19-resources/ covid-19-bsti-reporting-templates/. Accessed 4 Jul 2020

11. Borghesi A, Maroldi R (2020) COVID-19 outbreak in Italy: experimental chest $\mathrm{X}$-ray scoring system for quantifying and monitoring disease progression. Radiol Med 125:509-513. https://doi.org/10. 1007/s11547-020-01200-3

12. Kaleemi R, Hilal K, Arshad A et al (2021) The association of chest radiographic findings and severity scoring with clinical outcomes in patients with COVID-19 presenting to the emergency department of a tertiary care hospital in Pakistan. PLoS One 16:e0244886. https://doi.org/10.1371/journal.pone.02448 86

13. Balbi M, Caroli A, Corsi A et al (2021) Chest X-ray for predicting mortality and the need for ventilatory support in COVID19 patients presenting to the emergency department. Eur Radiol 31:1999-2012. https://doi.org/10.1007/s00330-020-07270-1
14. Schalekamp S, Huisman M, van Dijk RA et al (2021) Model-based prediction of critical illness in hospitalized patients with COVID19. Radiology 298:E46-E54. https://doi.org/10.1148/radiol.20202 02723

15. Kerpel A, Apter S, Nissan N et al (2020) Diagnostic and prognostic value of chest radiographs for COVID-19 at presentation. West J Emerg Med 21:1067-1075. https://doi.org/10.5811/westj em.2020.7.48842

16. Mesas AE, Cavero-Redondo I, Álvarez-Bueno C et al (2020) Predictors of in-hospital COVID-19 mortality: a comprehensive systematic review and meta-analysis exploring differences by age, sex and health conditions. PLoS One 15:e241742. https://doi.org/ 10.1371/journal.pone.0241742

17. Wong HYF, Lam HYS, Fong AH-T et al (2020) Frequency and distribution of chest radiographic findings in patients positive for COVID-19. Radiology 296:E72-E78. https://doi.org/10.1148/ radiol.2020201160

Publisher's note Springer Nature remains neutral with regard to jurisdictional claims in published maps and institutional affiliations. 